\title{
Relación entre sociabilidad, presión arterial y frecuencia cardiaca en el perro doméstico (Canis familiaris)
}

\author{
RELATION BETWEEN SOCIABILITY, BLOOD PRESSURE AND HEART RATE IN THE DOMESTIC DOG \\ (CANIS FAMILIARIS)
}

\author{
Romina Cainzos $^{1,2}$, María Belén Delgado ${ }^{1}$, Patricia Koscinczuk ${ }^{1}$
}

\section{Resumen}

El objetivo del estudio fue evaluar la frecuencia cardiaca (FC) y la presión arterial (PA) en perros domésticos de un criadero como respuesta al contacto con una persona desconocida, teniendo en cuenta si la sociabilidad influye en estas respuestas. Se trabajó con 18 perros adultos, entre 1 a 8 años, de ambos sexos, de las razas Smooth Fox Terrier, Wire Hair Fox Terrier y Beagle, de un criadero de la ciudad de Corrientes, Argentina. Se aplicó un test de sociabilidad que constaba de dos fases de dos minutos cada una. En la fase pasiva la persona no interactúa con el animal y en la fase activa la persona interactúa con el animal por medio de caricias. Se registraron las variables de latencia, contacto físico y visual y señales de miedo/sumisión. Con base a las respuestas, los animales se distribuyeron en un grupo poco social $(1 ; n=9)$ y uno social $(2 ; n=9)$. Se determinó la presión arterial (sistólica [PS], diastólica [PD], media [PM]) y la frecuencia cardiaca (FC) antes y después de la interacción con la persona desconocida. Los perros del grupo 2 (sociables) presentaron diferencia significativa en las mediciones de PS y FC antes y después de la interacción ( $\mathrm{p}=0.02$ y $\mathrm{p}=0.04$, respectivamente). Asimismo, se encontró diferencia significativa entre grupos para la variable PS después de la interacción con la persona $(\mathrm{p}=0.03)$. La interacción con la persona desconocida aumentó la FC y PS en los perros poco sociales, en tanto que disminuyó la PS en los perros sociales.

Palabras clave: caninos; hipertensión; comunicación social; relación humano animal; bienestar

\footnotetext{
${ }^{1}$ Cátedra de Patología Médica, Facultad de Ciencias Veterinarias, Universidad Nacional del Nordeste, Corrientes, Argentina

2E-mail:romicainzos@hotmail.com
}

Proyecto de investigación acreditado por la Secretaria General de Ciencia y Técnica, Corrientes, Argentina. Resolución N. ${ }^{\circ}$ 893/13 CS, Código: B014-2013

Recibido: 6 de marzo de 2017

Aceptado para publicación: 17 de octubre de 2017 
The aim of the study was to evaluate heart rate (HR) and blood pressure (BP) in domestic dogs of a breeding facility in the city of Corrientes, Argentina in response to contact with an unknown person, taking into account whether sociability influences these responses. A total of 18 adult dogs, aged 1 to 8 years, of both sexes, of the Smooth Fox Terrier, Wire Hair Fox Terrier and Beagle breeds were studied. A sociability test was applied, consisting of two phases of two minutes each. In the passive phase the person does not interact with the animal and in the active phase the person interacts with the animal through caresses. The variables of latency, physical and visual contact and signs of fear/submission were registered. Based on the responses, the animals were distributed in a less social group $(1, n=9)$ and a more social group $(2, n=9)$. Blood pressure (systolic [PS], diastolic [PD], mean [PM]) and heart rate (FC) were determined before and after interaction with the unknown person. Dogs of group 2 (sociable) showed significant differences in PS and FC measurements before and after the interaction ( $\mathrm{p}=0.02$ and $\mathrm{p}=0.04$, respectively). Likewise, there was a significant difference between groups for the PS variable after interaction with the person $(\mathrm{p}=0.03)$. The interaction with the unknown person increased the FC and PS in the less social dogs, while the PS decreased in social dogs.

Key words: canines; hypertension; social communication; human animal relationship; welfare

\section{INTRODUCCIÓN}

Los perros adultos desarrollan rápidamente un vínculo de apego con los seres humanos que interactúan con ellos, condición que se la conoce como sociabilidad. Se le puede definir como el proceso por el cual los individuos adquieren el conocimiento, las habilidades y las disposiciones que los capacita para participar de forma más o menos activa como miembros pertenecientes a grupos y a una sociedad concreta (Svartberg y Forkman, 2002).

El perro doméstico (Canis familiaris) comparte su ambiente cotidiano con las personas. Compartir las mismas condiciones ambientales puede llevar a conductas de señalización convergentes que facilitan la comunicación de especies no directamente relacionadas. En este sentido, en su convivencia con el hombre, el perro aprende a considerarlo como un miembro del grupo social. Para las especies sociales como el perro, el aprendizaje a través de los demás es esencial, debido a que, una gran parte de la interacción social y la cohesión se determinan por la correcta identificación de señales de comportamiento que facilitan la adaptación al medio ambiente. Los perros tienen una serie de habilidades cognitivas que les permite responder a las diferentes señales dadas por los seres humanos (Miklósi, 2009).

Estresores psicológicos son los estímulos naturales más potentes que afectan a la corteza suprarrenal y la pituitaria. Los perros son afectados por muchos de estos factores, especialmente cuando se presentan situaciones novedosas, impredecibles o incontrolables (Coppola et al., 2006; Morberg, 2000). Algunos factores de estrés específicos que influyen en el eje hipotalámico-pituitariosuprarrenal (HPA) incluyen la exposición a un entorno nuevo o amenazante, la separación de los objetos de apego, la imprevisibilidad de los eventos externos y la falta o pérdida de control sobre el ambiente (Coppola et al., 2006). 
Además, el aislamiento social o restricción es considerado también como un importante factor estresante para una especie social como el perro. Con el tiempo, la reducción de la interacción humano-animal puede dar lugar a la interrupción de la homeostasis fisiológica y conductual (Barrera et al., 2010).

El cerebro provoca respuestas de estrés que son acordes con la naturaleza del estímulo. Factores de estrés físico, tales como la pérdida de sangre, infección y dolor requieren de una reacción inmediata «sistémica» que se desencadena por mecanismos reflejos (Morberg, 2000). El cerebro responde también al estrés no físico o «psicógeno» basado en las experiencias previas o innatas. Estas respuestas están dadas por la activación del sistema nervioso autónomo (Shin y Shin, 2017; Bergamasco et al., 2010). Una de las manifestaciones sistémicas del estrés agudo es la hipertensión arterial. Si el estresor persiste en el tiempo puede provocar un estrés crónico, lo cual coloca al animal en un estado pre-patológico, pudiendo afectar el bienestar del individuo (Morberg, 2000). La exposición al estrés crónico altera la estructura física y la función de las regiones cerebrales involucradas en el control de HPA y las respuestas fisiológicas al estrés (Beerda et al., 1998; Bergamasco et al., 2010).

Algunos cambios medioambientales, como el aumento de interacción con humanos, tienen un efecto positivo sobre el comportamiento y el bienestar de los perros en cautiverio. El contacto con los seres humanos puede moderar o prevenir tanto, la activación del eje HPA, así como también, la respuesta autonómica a factores de estrés agudo (Bergamasco et al., 2010). Se ha demostrado que los perros pueden mostrar algunas respuestas relacionadas con el estrés en la interacción física con el humano, de allí que la importancia de identificar las interacciones específicas humano-animal que podría alterar el bienestar del perro (Jakovcevic et al., 2012). Es posible que los perros en cautiverio exhiban comportamientos de miedo hacia los seres humanos (Pullen et al., 2012). Por otro lado, estudios anteriores han indicado que la interacción humana puede aliviar el estrés de los perros alojados en un ambiente con restricción social (Coppola et al., 2006).

La activación del sistema nervioso simpático se puede utilizar como indicador etológico, fisiológico y hormonal para evaluar y monitorear el bienestar de los animales (Bergamasco et al., 2010). Las medidas no invasivas de evaluación de los parámetros fisiológicos en perros expuestos a actividades diferentes y desafíos ambientales comprenden mayormente los niveles salivales de cortisol, presión arterial, frecuencia respiratoria y frecuencia cardiaca (Kuhne et al., 2014b).

Las respuestas de los perros a las interacciones hombre-animal dependen del desarrollo del perro (por ejemplo, experiencias durante el periodo de socialización) y de la situación particular (por ejemplo, la atención física por parte de las personas) (Barrera et al., 2010; Pullen et al., 2012). Algunos autores han demostrado que es muy probable que las diferencias en los comportamientos de sociabilidad puedan estar relacionadas con situaciones interactivas más frecuentes con personas desconocidas en perros alojados en cautiverio, en comparación con los perros de familia (Shin y Shin, 2017). La exposición a un ser humano desconocido se utiliza comúnmente en la evaluación del comportamiento de los perros alojados en cautiverio, ya sea en refugio o criadero. En algunos estudios, el experimentador muestra un comportamiento indiferente o pasivo, mientras que en otros, muestra un comportamiento proactivo hacia el individuo, llamando al animal por su nombre y acariciándolo (Barrera et al., 2010).

El objetivo de este estudio fue evaluar las respuestas de frecuencia cardiaca y de presión arterial en perros pertenecientes a un criadero al contacto físico con un humano desconocido, teniendo en cuenta si la sociabilidad de los animales influye en estas respuestas. 


\section{Materiales y Métodos}

Entre marzo y junio de 2016 se trabajó con 18 perros adultos, clínicamente sanos, entre 1 y 8 años, de ambos sexos, de las razas Smooth Fox Terrier (pelo liso), Wire Hair Fox Terrier (pelo duro) y Beagle. Los animales pertenecían a un criadero de la Ciudad de Corrientes Capital (Argentina), inscrito en la Federación Cinológica Internacional (FCI). Los perros vivían en grupos de a dos, en caniles de $5 \times 1.50 \mathrm{~m}$. Cada canil tenía una zona de descanso, protegida con techo y paredes, y otra de recreo al aire libre. Los caniles estaban separados por una malla de alambre que permitía el contacto visual y olfativo entre los animales. Se disponía de comederos individuales en la zona de descanso, en tanto que el agua se proveía en la zona de recreo.

El nivel de sociabilidad de los perros con el humano fue evaluado con el test validado por Bentosela y Mustaca (2007), basado en los trabajos de Svartberg y Forkman (2002). La prueba se realizó en una habitación cerrada de $9 \mathrm{~m}^{2}$. Los animales fueron filmados durante 4 min con una cámara Sony (Cybershot 1080 HD, DSC-W230) colocada en una ventana que da hacia la habitación, a modo de cámara de Gesell. Se permitió a los perros explorar el lugar durante 5-10 min previo al inicio de la prueba. Esta se dividió en una fase pasiva y otra activa, cada una de 2 min. En la fase pasiva, una persona desconocida para el animal se encuentra sentada en una silla leyendo, sin interactuar con el animal (Figura 1a). En la fase activa, la persona se para y llama al animal por su nombre, y si este se acerca, la persona interactúa con el animal mediante caricias (Figura 1b). Esta persona, de sexo femenino, fue la misma en todos los casos y era desconocida por los animales. Se registraron las variables de sociabilidad como latencia (segundos), contacto físico (frecuencia de ocurrencia) y contacto visual (frecuencia de ocurrencia).

Se considera como 'latencia' el tiempo que tarda el animal en acercarse a la perso na desconocida. Esta variable se registró solo durante la fase pasiva. El tiempo de latencia fue medido por medio de un cronometro digital de pulsera. El contacto físico es la cantidad de veces que el animal toca a la persona con el hocico o con las patas. El contacto visual es la cantidad de veces que el animal mira a la cara de la persona. Además, se registraron señales de miedo-sumisión (orejas bajas, cola baja, posición de agazapado). Estas variables se evaluaron en las dos fases de la prueba.

Teniendo en cuenta que la sociabilidad es la tendencia a acercarse e interactuar con una persona u otros perros, se denominó perros menos sociales (grupo 1) a aquellos individuos cuya latencia fue superior a $50 \mathrm{~s}$, con una frecuencia de contacto táctil y visual menor o igual a 3 veces, y que hayan mostrado al menos dos de las tres señales de miedo-sumisión. Se consideró como perros más sociales (grupo 2) a aquellos individuos cuya latencia fue inferior a $\operatorname{los} 50 \mathrm{~s}$, con frecuencia de contacto táctil y visual superior a 3 veces, y mostraron una o ninguna de las tres señales de miedo-sumisión (Cuadro1).

Con base a los resultados, el grupo 1 estuvo conformado por nueve perros $(3.4 \pm$ 1.4 años) y el grupo 2 con nueve perros (4.0 \pm 2.4 años). A cada animal se le tomó la presión arterial (mmHG) y frecuencia cardiaca (lat/min) por el método oscilométrico, mediante el uso de un tensiómetro de uso veterinario V6Vet (Veterinary Clinical Monitor ${ }^{\circledR}$ ), antes y después de la interacción con la persona desconocida. Se registraron las variables de presión sistólica (PS), diastólica (PD), media (PM) y frecuencia cardiaca (FC). La medición de la presión arterial se realizó en la misma habitación en donde se realizó la prueba de interacción y luego que el animal tuvo tiempo suficiente de familiarizarse con el ambiente, pero antes de medir la frecuencia cardiaca. La habitación permaneció cerrada durante la medición, y en ausencia de otros perros, personas y ruidos de fondo. 


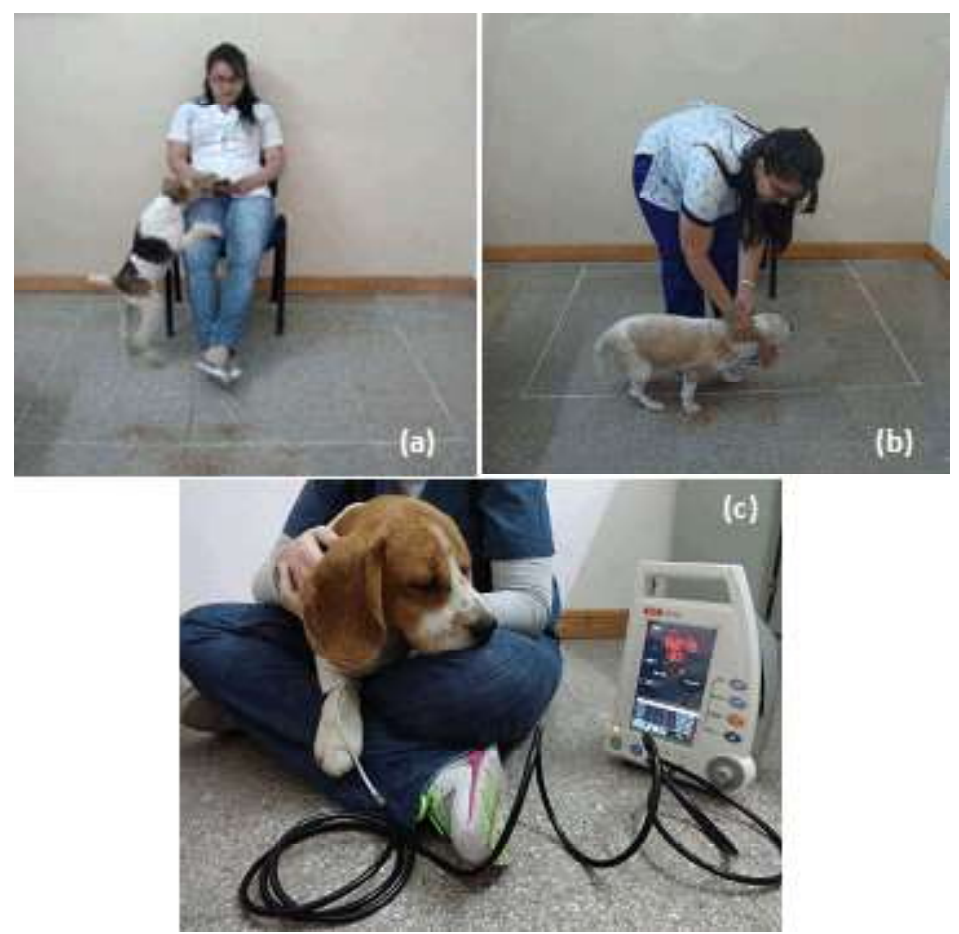

Figura 1. Prueba de socialización. (a). Primera fase donde la persona permanece sentada en la silla y el animal realiza el contacto con ella. (b) Segunda fase donde la persona interactúa con el animal. (c) Toma de la presión arterial

Cuadro 1. Valores (media \pm d.e.) de las variables de sociabilidad de 18 perros, según nivel de sociabilidad frente a una persona desconocida

\begin{tabular}{lccc}
\hline Grupos & $\begin{array}{c}\text { Promedio de } \\
\text { latencia }\end{array}$ & $\begin{array}{c}\text { Promedio de } \\
\text { contacto táctil }\end{array}$ & $\begin{array}{c}\text { Promedio de } \\
\text { contacto visual }\end{array}$ \\
\hline $\begin{array}{l}\text { Grupo 1 } \\
\text { Baja sociabilidad }\end{array}$ & $0.6 \pm 0.1$ & $1.8 \pm 1.0$ & $1.5 \pm 0.6$ \\
$\begin{array}{l}\text { Grupo2 } \\
\text { Alta sociabilidad }\end{array}$ & $0.3 \pm 0.1$ & $4.8 \pm 1.0$ & $4.8 \pm 1.0$ \\
\hline
\end{tabular}

La medición de la presión arterial fue realizada por una persona diferente a la que realizó el test de interacción, que conocía a los animales y que tenía conocimientos en la técnica de medición. Para la toma de presión arterial colocó el animal sobre su regazo para facilitar el relajamiento de los perros. Para minimizar aún más los efectos de error en la medición, se seleccionaron los manguitos te- niendo en cuenta un tamaño entre 30 y $40 \%$ de la circunferencia de la extremidad de cada sujeto evaluado. En este trabajo, el manguito fue colocado en el miembro anterior derecho con coincidencia con la arteria braquial (Figura 1c). Se realizaron cuatro mediciones por cada animal, descartándose la primera por arrojar valores extremos, y promediando los tres valores siguientes. 
Cuadro 2. Valor $\mathrm{P}$ para muestras apareadas de la presión sistólica y de la frecuencia cardiaca del grupo de perros más sociables (grupo 2) después de la presencia de una persona desconocida

\begin{tabular}{lccc}
\hline Variables & Media & $\begin{array}{c}\text { Valor } \\
\mathrm{T}\end{array}$ & $\begin{array}{c}\text { Valor } \\
\mathrm{P}\end{array}$ \\
\hline $\begin{array}{l}\text { Presión } \\
\text { sistólica }\end{array}$ & 8.11 & 2.79 & 0.0234 \\
$\begin{array}{l}\text { Frecuencia } \\
\text { cardiaca }\end{array}$ & 8.22 & 2.41 & 0.0423 \\
\hline
\end{tabular}

Las variables PS, PD, PM y FC mostraron una distribución normal en ambos grupos (Shapiro-Wilks modificado), de modo que se aplicó la prueba de $\mathrm{T}$ para muestras apareadas, fijando un valor de alfa $=0.05$, para comparar las variables dentro de cada grupo. La comparación entre ambos grupos se hizo mediante prueba de $\mathrm{T}$ para muestras independientes.

El estudio fue aprobado por el comité de Ética y Bioseguridad de la Facultad de Ciencias Veterinarias de la Universidad Nacional del Nordeste, Corrientes, Argentina (Resolución N. ${ }^{\circ}$ 471/2009-CD).

\section{Resultados}

En el análisis de la prueba de $\mathrm{T}$ para muestras apareadas se encontraron diferen- cias significativas para las variables PS y FC en el grupo 2 (Cuadro 2). Antes de la interacción con la persona, los valores de PS y FC fueron de $120.1 \pm 15.0 \mathrm{mmHg}$ y de $98.0 \pm 17.0 \mathrm{lat} / \mathrm{min}$, respectivamente, descendiendo a $112.0 \pm 18.3 \mathrm{mmHg}$ y $89.8 \pm 22.3$ lat/min, respectivamente, luego del contacto con la persona. En cambio, en el grupo 1 no se observaron diferencias significativas para ninguna de las variables en estudio.

La prueba de T para muestras independientes indicó diferencia significativa para la variable PS después de la interacción con una persona desconocida $(\mathrm{p}=0.03)$ en ambos grupos de perros. La media aritmética de PS fue más elevada después de la interacción con un humano desconocido en los perros del grupo 1 (antes: $124.7 \pm 24.3 \mathrm{mmHg}$; después: $132.6 \pm 18.9 \mathrm{mmHg}$ ), en comparación con los perros del grupo 2 (antes: $120.1 \pm 15.1$ mmHg; después: $112.0 \pm 18.3 \mathrm{mmHg}$ ) (Cuadro 3).

\section{Discusión}

Los perros son animales sociales, lo que significa que, al igual que las personas, viven en grupo. La socialización es el proceso mediante el cual se aprende el comportamiento social apropiado y, en general, se refiere a la socialización primaria con miembros de la misma especie, así como de otras especies (Bentosela y Mustaca, 2007; Jakovcevic et al., 2012).

Cuadro 3. Valores (media aritmética) de la presión arterial y frecuencia cardiaca en perros antes (A) y después (D) de la interacción con una persona desconocida

\begin{tabular}{lcccccccc}
\hline & PS A & PS D & PD A & PD D & PM A & PM D & FC A & FC D \\
\hline Grupo 1 & 124.7 & 132.6 & 63.4 & 70.8 & 81.0 & 91.4 & 94.3 & 89.9 \\
Grupo 2 & 120.1 & 112.0 & 78.6 & 74.4 & 94.8 & 85.3 & 98.0 & 89.8 \\
\hline
\end{tabular}

PS: Presión sistólica; PD: Presión diastólica; PM: Presión media; FC: Frecuencia cardiaca 
Es por ello que los perros con alto nivel de sociabilidad pueden estar más dispuestos a interactuar con la gente y son más persistentes y resistentes a la extinción de las respuestas aprendidas. Asimismo, es posible que la presencia del humano que realiza contacto visual con el perro actúe como ser social reforzador, manteniendo así la respuesta comunicativa (Bentosela y Mustaca, 2007). En este estudio se encontró que los perros considerados como sociales realizaron mayor frecuencia de contacto (táctil y visual) con la persona desconocida.

Es conocido que la socialización con otros perros y seres humanos es esencial para el bienestar psicológico del perro. De hecho, se cree que el contacto humano puede ser más importante que el contacto con otro perro (Coppola et al., 2006). Por otro lado, Pullen et al. (2012) han propuesto que la familiaridad hacia la persona no afecta los niveles de juegos en perros bien socializados, los perros permanecen receptivos al contacto humano independientemente de si están o no familiarizados con la persona, mientras que para perros menos sociales la presencia de una persona desconocida podría actuar como una amenaza o como un factor de estrés (Coppola et al., 2006; Jakovcevic et al., 2012). En el presente estudio, la presencia de una persona desconocido pudo haber generado una respuesta de estrés en los perros considerados como menos sociales.

De la misma forma, los perros más sociales estarían más atentos a las personas y pueden aprender sus señales con más facilidad y responder más eficazmente a sus órdenes durante la formación y el desempeño real (Bentosela y Mustaca, 2007). Cuanto mayor sea el comportamiento social que exhibe un animal, más exitoso puede ser el vínculo entre el ser humano y el animal. (Odendaal y Meintjes, 2003).

En función de cómo fue socializado un animal cuando era joven y de las experiencias que tenga con las personas en cualquier etapa de su vida, puede generar miedo a los individuos a los que no están acostumbrados o a los que asocia con una experiencia aversiva (Barrera et al., 2012). El miedo es una emoción que induce una respuesta adaptativa, que permite al animal evitar situaciones que podrían ser peligrosas. Esta respuesta se inicia cuando el animal percibe un estímulo que interpreta como potencialmente perjudicial que causa el principio de una respuesta de estrés (Moberg y Mench, 2000), y este estímulo podría ser el contacto con una persona desconocida. En los perros socializados a los seres humanos y adaptados a su sistema social, probablemente estas respuestas se reducen, en presencia de un ambiente novel o desconocidos para ellos (Coppola et al., 2006); en cambio, los perros menos sociales pueden llegar a sufrir altos niveles de estrés en situaciones desconocidas como el abandono, traslado a otro lugar, visita al veterinario y restricción social, entre otras (Shin y Shin, 2017).

El aislamiento social o restricción se considerado también como un importante factor de estrés para una especie social como el perro (Bergamasco et al., 2010). Perros que se encuentran en condiciones de aislamiento físico y social pueden padecer de trastornos del comportamiento, tales como alta frecuencia de auto-aseo, dar vueltas, postura agazapada, suspiros y coprofagia (Bergamasco et al., 2010; Barrera et al., 2012). En este estudio, todos los animales provenían de un criadero donde había contacto entre perros y estos mantenían escaso contacto con seres humanos, con excepción de dos personas que les daban de comer y les limpiaban los caniles. Es así, que se podría suponer que la falta de contacto con más personas y viviendo en un ambiente pobremente enriquecido podría haber afectado el comportamiento de los animales del estudio.

Es sabido que el cerebro también responde al estrés no físico o «psicógeno», basado en la experiencia previa o innata del animal. Estas repuestas pueden ocurrir en anticipación a un estímulo o en relación a los 
acontecimientos estresantes (Ulrich-Lai y Herman, 2009). Los animales tienden a mostrar señales de ansiedad, de miedo o sumisión cuando son expuestos a situaciones nuevas (Pullen et al., 2012). En este estudio, estas señales se vieron en aquellos perros con bajo nivel de sociabilidad al colocarlos frente a una persona desconocida. Estas señales se generan mediante la activación del sistema nervioso autónomo como respuesta inmediata a la exposición de un estresor, a través del aumento de la actividad simpática y disminución de la actividad parasimpática. La respuesta fisiológica al estrés consiste en mantener la integridad fisiológica, incluso en las circunstancias más exigentes. Estas respuestas provocan un incremento de la presión arterial ante la liberación de adrenalina (por estimulación directa de la medula adrenal) y noradrenalina (desde los nervios simpáticos) (Ulrich-Lai y Herman, 2009; Bergamasco et al., 2010). A mayor sean los gestos de apaciguamiento mayor es su actividad simpática (Kuhne et al., 2014a).

Debido a que la presión arterial es sensible al estrés psicológico, los perros sensibles al estrés presentan presiones sanguíneas más elevadas. Los individuos son capaces de variar su respuesta cardiovascular según en el medio ambiente que se encuentran (Vincent y Michell, 1995). Es por ello que en este estudio se pudo observar que perros menos sociales sometidos a la interacción con una persona desconocida mostraron un aumento de la presión arterial después de dicha interacción, posiblemente como resultado de una respuesta rápida de estrés psicológico.

Las caricias pueden tener efectos variables en el perro, dependiendo del apego existente entre el perro y el hombre, y el contexto de la actividad (Bergamasco et al., 2010; Kuhne et al., 2014a). Otros estudios neurofisiológicos dan apoyo a esta idea, mostrando que el contacto positivo entre el due- ño y el perro tiene propiedades ansiolíticas para ambos, resultando en una disminución de la presión sanguínea después de una interacción positiva (Kuhne et al., 2014a). Los resultados indicaron que se puede lograr una disminución significativa de la presión sanguínea entre 5 y 24 minutos de interacción interespecífica positiva, tanto en humanos como en perros (Odendaal y Meintjes, 2003).

Asimismo, las variaciones de la FC están muy relacionadas con el estado emocional de los individuos, donde un estado emocional positivo produce una disminución de la FC (Katayama et al., 2016), aspecto que ocurrió en los perros más sociables. Las variaciones de la frecuencia cardiaca es un buen indicador para evaluar la actividad del sistema nervioso autónomo en respuesta a estrés psicofisiológicos modulados por el estado emocional (Katayama et al., 2016). En animales de granja y caballos se utilizan alteraciones cardiacas como marcador del estrés físico y mental, asumiendo que el aumento de la emotividad y el nivel de estrés se reflejan en un aumento de la FC (Bergamasco et al., 2010). Por lo tanto, la disminución de la FC observada en los animales del grupo 2 podría reflejar un bajo nivel de estrés. A su vez, estudios previos han demostrado que los perros muestran una actividad motora mínima y una disminución de la frecuencia cardiaca mientras son acariciados por una persona desconocida (Kuhne et al., 2014a). Una sesión de contacto positivo con un humano puede influir en la disminución de la respuesta al estrés (Kuhne et al., 2014b).

\section{Conclusión}

Se pudo observar que la presión arterial y la frecuencia cardiaca disminuyen en los perros considerados como más sociales después de la interacción con una persona desconocida. 


\section{Literatura Citada}

1. Barrera G, Giamal Y, Mustaca A, Bentosela M. 2012. Relación entre el tipo de alojamiento y las respuestas de mirada, sociabilidad y miedo-apaciguamiento en perros. Suma Psicológica 19(2): 7-18.

2. Barrera G, Jakovcevic A, Elgier A M, Mustaca A, Bentosela M. 2010. Responses of shelter and pet dogs to an unknown human. J Vet Behav Clin Applic Res 5:339-344.

3. Beerda B, Schilder MB, van Hooff $J A$, de Vries HW, Mol JA. 1998. Behavioural, saliva cortisol and heart rate responses to different types of stimuli in dogs. Appl Anim Behav Sci 58: 365-381. doi: 10.1016/S0168-1591(97)00145-7

4. Bentosela M, Mustaca A. 2007. Comunicación entre perros domésticos (canis familiaris) y hombres. Rev Latinoam Psicol 39: 375-387.

5. Bergamasco L, Osella MC, Savarino P, Larosa G, Ozella L, Manassero M, Badino $P$, et al. 2010. Heart rate variability and saliva cortisol assessment in shelter dog: human-animal interaction effects. Appl Anim Behav Sci 125: 5668. doi: 10.1016/j.applanim.2010.03.002

6. Coppola CL, Grandin T, Enns RM. 2006. Human interaction and cortisol: can human contact reduce stress for shelter dogs? Physiol Behav 87: 537-541. doi: 10.1016/j.physbeh.2005.12.001

7. Jakovcevic A, Mustaca A, Bentosela M. 2012. Do more sociable dogs gaze longer to the human face than less sociable ones? Behav Process 90: 217-222. doi: 10.1016/j.beproc.2012.01.010

8. Katayama M, Kubo T, Mogi K, Ikeda K, Nagasawa M, Kikusui T. 2016. Heart rate variability predicts the emotional state in dogs. Behav Process 128: 108-112. doi: 10.1016/j.beproc. 2016.04.015
9. Kuhne F, Hößler JC, Struwe R. 2014a. Behavioral and cardiac responses by dogs to physical human-dog contact. J Vet Behav Clin Appl Res 9: 93-97. doi: 10.1016/j.jveb.2014.02.006

10. Kuhne F, Hößler JC, Struwe R. $2014 b$. Emotions in dogs being petted by a familiar or unfamiliar person: Validating behavioural indicators of emotional states using heart rate variability. Appl Anim Behav Sci 161: 113-120. doi: 10.1016/ j.applanim.2014.09.020

11. Miklósi A. 2009. Social cognition. In: Dog behaviour, evolution, and cognition. $3^{\text {rd }}$ ed. Oxford: University Press. p 165200.

12. Morberg GP. 2000. Biological responses to stress: implications for animal welfare. In: Morberg GP, Mench JA (eds). The biology of animal stress. Basic principles and implications for animal welfare. UK: CABI Publishing. p 1-21.

13. Morberg GP, Mench JA. The biology of animal stress. Basic principles and implications for animal welfare. UK: CABI Publishing. $377 \mathrm{p}$.

14. Odendaal JS, Meintjes RA. 2003. Neurophysiological correlates of affiliative behaviour between humans and dogs. Vet J 165: 296-301. doi: 10.1016/S1090-0233(02)00237-X

15. Pullen AJ, Merrill RJN, Brasdshaw JWS. 2012. The effect of familiarity on behavior of kennel dogs during interactions with humans. Appl Anim Behav Sci 137: 66-73. doi: 10.1016/ j.applanim.2011.12.009

16. Shin YJ, Shin NS. 2017. Relationship between sociability toward humans and physiological stress in dogs. J Vet Med Sci 79: $1278-1283$. doi: 10.1292/jvms.160403

17. Svartberg K, Forkman B. 2002. Personality traits in the domestic dog (Canis familiaris). Appl Anim Behav Sci 79: 133-155. doi: 10.1016/S01681591(02)00121-1 
18. Ulrich-Lai YM, Herman JP. 2009. Neural regulation of endocrine and autonomic stress responses. Nat Rev Neurosci 10: 397-409. doi: 10.1038/ nrn2647
19. Vincent IC, Michell AR. 1995. Relationship between blood pressure and stress-prone temperament in dogs. Physiol Behav 60: 135-138. doi: 10.1016/ 0031-9384(95)02273-2 